Cahiers $d u$ MONDE RUSSE

\section{Cahiers du monde russe}

Russie - Empire russe - Union soviétique et États indépendants

$59 / 4 \mid 2018$

Varia

\title{
Dan Healey, Russian Homophobia from Stalin to Sochi
}

\section{Arthur Clech}

\section{OpenEdition \\ Journals}

Édition électronique

URL : https://journals.openedition.org/monderusse/10832

DOI : 10.4000/monderusse.10832

ISSN : 1777-5388

Éditeur

Éditions de l'EHESS

Édition imprimée

Date de publication : 1 octobre 2018

Pagination : 687-693

ISBN : 978-2-7132-2747-9

ISSN : $1252-6576$

\section{Référence électronique}

Arthur Clech, «Dan Healey, Russian Homophobia from Stalin to Sochi », Cahiers du monde russe [En ligne], 59/4 | 2018, mis en ligne le 01 octobre 2018, consulté le 07 janvier 2022. URL : http:// journals.openedition.org/monderusse/10832 ; DOI : https://doi.org/10.4000/monderusse.10832 


\section{Dan HEALEY}

\section{Russian Homophobia from Stalin to Sochi}

London - New York : Bloomsbury Academic, 2017, 312 p., 18 ill.

Écrire une histoire de l'« homophobie russe » de Stalin à nos jours a tout d'un défi et Dan Healey le relève sans se départir du souci permanent de rendre compte de l'homophobie contemporaine en Russie. Il s'inscrit dans une perspective historique et ne tombe pas dans l'écueil de l'homonationalisme, même quand, dans sa préface, il dénonce les exactions de Ramzam Kadyrov contre des Tchétchènes accusés d'être gays. Au contraire, en tant qu'historien, il propose de contribuer à un travail de mémoire, qui est obstrué par le récit mythique d'une Russie « innocente » et hétérosexuelle, hérité de l'époque soviétique et impériale. Une telle analyse permet de mieux saisir la vulgate officielle d'une homophobie d'État qui a choisi habilement ses mots et les a inscrits dans une loi adoptée en 2013 punissant de sévères amendes « toute propagande des relations non traditionnelles » auprès des mineurs. Cette histoire ne saurait être exhaustive, précisément parce qu'une entreprise nationale d' " amnésie » " russe » est déployée pour exclure ce que l'historien appelle les « queers », terme choisi pour inclure tant les lesbiennes, les gays que les bisexuels et les bisexuelles, les intersexes ou les trans ${ }^{1}$.

Histoire politique avant tout, ce travail historique constituera sans nul doute un véritable guide de la répression médico-légale des « queers » soviétiques et russes. Sans se soumettre complètement au récit chronologique suggéré par le titre de l'ouvrage (1934 : réintroduction de l'article de loi anti-sodomie - 2014 : Jeux Olympiques de Soči), la structure de l'ouvrage est conçue de sorte à stimuler une réflexion en croisant brillamment différents appareils critiques théoriques tout en offrant une compréhension de l'histoire soviétique et de l'histoire de la Russie contemporaine. Cette structure ne néglige pas pour autant l'état des lieux de la recherche que l'auteur établit scrupuleusement, pour chaque période étudiée, jusqu'à tenir compte explicitement de travaux non encore publiés lors de la rédaction de l'ouvrage.

Ainsi, pour débuter, Dan Healey choisit de retracer l'histoire encore méconnue de l'homosexualité au goulag, la partie sans doute la plus violente de « l'homophobie russe ». Deux illustrations exposant des tatouages servent le propos. Dan Healey explique que de nombreux hommes dits « dégradés » au goulag sont réduits à un esclavage sexuel par d'autres hommes, l'acte du viol consacrant l'identité du stigmatisé. Environ mille hommes par an étaient incarcérés sous l'article de loi anti-sodomie. Sachant que le nombre de condamnations allait croissant pour l'époque soviétique tardive ${ }^{2}$, ces hommes ne constituaient donc qu'une partie seulement des « dégradés » du goulag. Cependant, l'auteur choisit comme illustration le tatouage infamant d'un détenu sous le chef de l'article de loi anti-sodomie, réintroduit par Stalin en 1934 et aboli seulement en 1993. Le second tatouage, celui d'une femme lesbienne dite « passive » sur laquelle est inscrit l'œil de sa partenaire sexuelle « active », montre dans quelle mesure le milieu carcéral soviétique a été le lieu d'une visibilité homosexuelle strictement agencée selon une hiérarchie pyramidale. De crainte que des pratiques homosexuelles ne se répandent avec le retour 
de nombreux détenus des camps sous Hruščev, le régime renforce les persécutions des homosexuels qui présentent un volet pénal mais aussi un volet psychiatrique, la psychiatrisation frappant les hommes comme les femmes. L'historien tire profit de ses recherches dans le domaine de la médecine légale soviétique, notamment la médecine au goulag, pour documenter et analyser des discours d'experts médicaux, mais aussi pour contextualiser la recrudescence de la pathologisation de l'homosexualité en URSS, puis celle à laquelle on assiste récemment dans la Russie contemporaine malgré la dépathologisation de 1999. En l'absence de discours publics sur l'homosexualité, sinon une « propagande homophobe » orchestrée par l'administration des camps sous Hruščev, se répandent des représentations particulièrement négatives de l'homosexualité, masculine comme féminine, associées à la violence des camps et du monde carcéral soviétique.

Nulle part ailleurs, on ne trouvera une description aussi dense et complète des hiérarchies sexuelles à l'œuvre dans le système carcéral soviétique qui nourrira un « dégoût » ${ }^{3}$ associé à l'homosexualité, fondement même de «l'homophobie russe », et qui a pu être relayé par les prisonniers " politiques », souvent membres de l'intelligentsia. Cette dernière déployait des stratégies sociales de distinction, mais aussi de survie en fonction des rapports de forces complexes en place dans le monde concentrationnaire soviétique. En guise de légitimation morale, voire religieuse de soi, l'intelligentsia articule un discours " homophobe » sur l'homosexualité qui est identifiée à une propriété sociale : elle serait inhérente aux droits communs, au lumpenproletariat dont le régime stalinien espérait la rapide réhabilitation sociale. Ainsi, l'auteur évoque des femmes lesbiennes qui, pour vivre leur désir homosexuel, doivent cacher dans les camps leur appartenance passée à l'intelligentsia. Certains membres de l'intelligentsia, homosexuels, ont pu trouver refuge dans les théâtres existant dans les camps avant qu'ils deviennent l'objet d'une surveillance particulière par l'administration. La crainte d'être « dégradé » - le « dégradé » occupant la place du paria, du pestiféré à l'écart des autres prisonniers ${ }^{4}-$ par d'autres détenus a renforcé une homophobie où ledit « actif » n'était pas considéré comme homosexuel. Or, cette homophobie cultivée dans le milieu carcéral a aussi contribué à prôner des canons de masculinité valorisant l'agressivité hors du camp ou de la prison. L'historien pointe d'ailleurs la nécessité d'approfondir une histoire de la masculinité russe pour l'époque soviétique et après.

$\mathrm{Si}$, pour cet ouvrage, l'auteur réaffirme que la « persécution par les experts et l'État des sujets queers » constitue son principal objet de recherche, il n'en développe pas moins d'autres niveaux d'analyse. Ainsi, on lui saura gré d'avoir accordé une place plus grande aux subjectivités « queer ». Il consacre notamment un chapitre au journal intime d'un chanteur célèbre condamné à deux reprises pour son homosexualité (1944, 1959), Vadim Kozin (1903-1994). L'historien aborde un discours à la première personne qui relate dix-huit mois (1955-1956) d'une tournée sibérienne du chanteur. Dense et toujours pertinente, l'analyse qu'il en donne, permet de saisir une situation unique pour l'URSS : hors du camp, la visibilité homosexuelle n'était guère concevable : la célébrité de cet homme et, partant, de sa condamnation, le livre ouvertement à la vindicte de ceux qui se sentent autorisés à le conspuer. 
Une étude de la subjectivité homosexuelle de cet homme aurait pu cependant être développée davantage. Il me semble que c'est le fait même que l'homosexualité de Kozin ait été rendue publique (predana oglaske), mise à l'index, qui inscrit l'homosexualité dans le registre de l'infamie ou de l'opprobre («pozor ») plutôt que dans celui de la « honte » (styd), vécue à l'aune individuelle. En écho avec d'autres témoignages, son discours confirme le peu de pertinence que la notion d'identité homosexuelle pouvait avoir dans le contexte soviétique. La logique d'affirmation de soi, susceptible de libérer un sentiment de honte intériorisé par une histoire chrétienne que la psychanalyse et la psychiatrie ont accompagnée, appartient à une histoire « occidentale » de la sexualité, l'affirmation de soi passerait peut-être par d'autres ressorts dans les années 1920 pour apparaître en tout cas inarticulable dans un discours dès l'époque stalinienne. Aucune identité comme aucune communauté ne pouvaient être revendiquées publiquement, l'une comme l'autre ne pouvaient donc pas faire l'objet d'un retournement stratégique, de la honte à la fierté homosexuelle. Il est éloquent de constater qu'aucune intériorisation de la honte (styd) n'est perceptible dans le journal intime de Kozin. Il s'agit bien d'autre chose : outre la condamnation pénale qu'il juge injuste, Kozin rapporte qu'il est en prise à un nouveau jugement, celui de l'opinion publique. Jusqu'à la période du dégel au moins, Oleg Kharkhodin a pu analyser comment le « collectif» œuvrait à « démasquer » les défections d'un individu : « dénoncer » et se dénoncer dispensaient donc les individus d'une inquisition qui se serait opérée à un niveau individuel $^{6}$. Précisément, Kozin ne témoigne dans son journal intime que du rapport des autres à lui-même : l'opprobre y apparaît de façon hégémonique, et il le déjoue en démasquant l'hypocrisie de ses contemporains. Fait notable, il rejette la pathologisation à laquelle l'homosexualité était alors identifiée, un mode de subjectivisation à même d'éprouver sa subjectivité homosexuelle (« les personnes qui vont lire ces lignes ne doivent pas penser qu'elles sont écrites par un schizophrène » p. 81). Par conséquent, son discours n'articule pas de rapport de soi à soi. Autrement dit, et cela est valable pour d'autres manières de se construire en tant que sujet en URSS, inférant aussi bien au statut social qu'à ladite « nationalité » inscrite dans les papiers d'identité, sa subjectivité homosexuelle s'élabore sur le mode de l'escapisme, de sorte à ne pas s'assigner d'identité, pour échapper à des logiques d'identification déployées par l'État soviétique, dont les effets d'atomisation restent actuels après la mort de Stalin?

En l'illustrant de photographies tirées de son travail dans les archives, l'auteur parvient, dans une écriture dense et dynamique, à incarner en un chapitre particulièrement remarquable deux études de cas, l'une se déroulant à l'époque du stalinisme tardif, l'autre à celle du dégel. Les faits se passent dans des lieux différents, mais dans des milieux semi-ruraux comparables, à proximité de Leningrad : « viol et sodomie à Nevdubstroi, 1946-1951 », et « Sodomie et meurtre à Rahia, 1955-1959 ». Ces études de cas permettent à l'auteur de décentrer l'analyse : il adopte moins la perspective d'une répression émanant directement de l'État pour s'inscrire pleinement dans l'écriture d'une histoire sociale. Une autre histoire apparaît, celle qui explore les silences entourant l'homosexualité, celle qui montre comment l'homosociabilité 
masculine contribuait à rendre l'homosexualité invisible. Ce chapitre constitue un apport essentiel sur la manière dont des subjectivités homosexuelles soviétiques, dans des milieux sociaux pourtant marqués par l'interconnaissance et une apparente homogénéité, prennent des formes différentes : entre visibilité et invisibilité, entre une certaine marginalisation sociale d'un célibataire et l'homosociabilité masculine d'hommes mariés. En témoigne par exemple la « visibilité » queer d'un homme qui se fait tuer par un homme avec lequel il avait des relations. Il semble avoir été tué non pas pour son désir, mais pour un gramophone, objet de convoitise certain. Comme l'analyse Dan Healey, des « hommes exploitent leur relation avec des femmes et avec des hommes efféminés " pour réaliser leur désir homosexuel. Impressionnant, car il multiplie les niveaux d'analyse, ce chapitre démontre notamment comment la catégorie de genre permet de rendre compréhensible la manière avec laquelle des hommes se créent des « espaces » et des « relations » homosexuels en tirant profit de leur privilège genré et en exerçant une violence qui ne l'est pas moins. La première étude de cas pose la question de l'invisibilité, tandis que la seconde constitue un témoignage inédit de la manière avec laquelle une visibilité a pu être vécue dans le quotidien par un homme qui parlait au féminin. Les perspectives de cette dernière étude de cas sont stimulantes et invitent à des approfondissements. Cet homme est perçu comme un « original » par ceux qui le côtoient (dans les réponses données à la police) et l'on s'interroge, par exemple, sur les conditions d'indulgence que permettait cette perception de l'« original », n'épousant ostensiblement ni le lexique de la pathologisation ni celui de la criminalisation dont l'homosexualité masculine faisait pourtant l'objet, une « indulgence » qui disparaît dès que l'homosexualité est identifiée dans une condamnation pénale, comme dans le cas évoqué de Vadim Kozin.

À la fin de son ouvrage, l'auteur aborde la délicate entreprise de rendre compte du désir homosexuel dans l'écriture des biographies et rappelle notamment que l'homosexualité de Čaïkovskij a été niée encore récemment en Russie. Dan Healey dénonce l'occultation de l'homosexualité de personnes célèbres par l'État (le silence du musée de Kozin de Magadan sur son homosexualité est éloquent), mais aussi par des universitaires. Il se réfère par exemple aux travaux de Nikolay Bogomolov et de John Malmstad qui, alors qu'ils font autorité sur la biographie de Mihail Kuzmin (1872-1936), sous-estimeraient la place qu'occupait la subjectivité homosexuelle de l'écrivain. Cela vaut aussi pour le poète Nikolaj Kljuev (1884-1937). Par ailleurs, Dan Healey évoque d'autres figures, moins connues, comme celle de la poétesse Anna Barkova (1901-1976), entrant en dissidence politique avec le régime, et envoyée à plusieurs reprises au camp. Cette figure pourrait d'ailleurs servir de pont où se croiserait enfin une certaine dissidence soviétique avec une « dissidente sexuelle».

La mémoire de ces figures « queer », de par la légitimité culturelle dont elles disposent, pourrait contribuer à une affirmation positive de soi en Russie par le truchement de l'identification. En offrant aussi une lecture historique et un rapport stratégique à la mémoire pour un objet qui ne bénéficie pas de la même légitimité culturelle, la pornographie gay russe par exemple, Dan Healey continue à chercher 
quelles stratégies sont mises en œuvre pour faire advenir une affirmation positive de soi. La pornographie gay russe a été commercialisée à la chute de l'URSS et sa production et son accès ont été facilités. L'apparition d'Internet à la fin des années 1990 en a favorisé le développement. De manière différenciée, selon les années 1990 ou 2000, des identités, gay et nationale, parviennent à se réconcilier en s'inscrivant dans un même scénario de films qui, précise l'historien, ne reproduisent pas toujours, pour les années 1990, les canons de beauté gays en vigueur dans la pornographie gay américaine de l'époque. Alors que le milieu carcéral constitue un des motifs érotiques de la pornographie gay occidentale, Dan Healey remarque l'absence du milieu carcéral soviétique dans la pornographie gay russe, signe qu'un certain passé soviétique ne passe pas. Le « cauchemar » de l' « homophobie russe », celui d'une hiérarchisation par l'humiliation de rapports sexuels subis, apparaît inappropriable. En revanche, l'armée soviétique, malgré la violence des bizutages $\left(\right.$ dedovšina $\left.{ }^{8}\right)$ peut être réinvestie aujourd'hui de manière positive par l'imaginaire sexuel gay russe. D'ailleurs, il l'est peut-être aussi pour prendre la forme d'une démonstration d'un sentiment d'appartenance à une identité « nationale », alors qu'une propagande « homophobe russe » s'échine à l'en exclure.

À juste titre, l'historien note que les hommes et les femmes vivant un désir homosexuel se constituent des espaces propres « invisibles », non seulement dans les espaces publics des grandes villes soviétiques des années 1960-1980, mais aussi dans des logements individuels dont l'accès s'élargit depuis Hruščev. Surtout, ils et elles seraient souvent conscients qu'une « libération » gay a lieu à l'Ouest. L'usage rigoureux de sources secondaires lui permet de reconstituer des traits saillants de l'époque soviétique tardive pour émettre l'hypothèse d'une affirmation de soi plus grande. Il retrouve aussi une citation ${ }^{9}$ confirmant les résultats de mon travail sur les subjectivités homosexuelles à l'époque soviétique tardive ${ }^{10}$. Sacha, ingénieur moscovite, aurait dit qu' « il est très difficile de développer l'idée d'une identité gay, et encore moins la conscience de notre oppression ». Tandis que de tels changements lui apparaissaient plus concevables en Pologne qu'en URSS, il ajoutait que si des « groupes de solidarité existent », « nous ne pouvons pas nous organiser à cause de la répression politique ». Il apparaît donc, me semble-t-il, que cet « Occident» connaissait des déclinaisons différentes : la rigidité « idéologique » des leaders de pays satellites soviétiques comme la RDA n'a pas empêché l'abolition de l'article anti-sodomie, avant que la RFA « occidentale » ne soit poussée, comme le note d'ailleurs Dan Healey, à l'entériner à son tour.

La force des discours religieux et l'effet qu'ils avaient sur la population avant 1917, durant l'époque soviétique, puis après l'effondrement de l'URSS restent peu étudiés pour l'(homo) sexualité et, comme le note Dan Healey, même les travaux de référence de Laura Engelstein pour l'époque prérévolutionnaire n'en ont pas restitué l'importance. On saura gré à l'historien d'avoir soulevé la question du religieux en montrant très clairement comment un discours orthodoxe homophobe apparaît après que le renouveau religieux a été finalement instrumentalisé par l'État russe contemporain. Dan Healey offre une contextualisation convaincante sur la manière dont la protection de l'enfance est invoquée à partir de la fin des années 
1990 pour suggérer toujours davantage un amalgame entre la pédophilie et l'homosexualité. Sans que le texte de loi fasse référence à la première, l'historien observe que cet amalgame est entretenu dans des déclarations publiques de manière significative. Dan Healey rend compte avec une rigueur implacable d'une analyse des discours publics condamnant l'homosexualité sans oublier non plus de brosser à grand trait l'histoire du militantisme « queer » soviétique et russe.

On se souvient d'un des apports majeurs de la première monographie de l'historien : ayant eu accès aux archives, il avait pu réfuter la thèse totalitarisante de Simon Karlinsky, un slaviste américain qui situait les sources de l' " homophobie » dans la révolution d'Octobre ${ }^{11}$. Mieux encore, il avait étayé sa thèse en démontrant le caractère intentionnellement politique de la décriminalisation de l'homosexualité pour les années 1920. S'il ne désavoue pas cet apport en inaugurant avec Stalin sa monographie sur l'" homophobie russe », son analyse tend à niveler des discours contradictoires sur la sexualité des années précédant le stalinisme en un seul discours bolchevik suspicieux sur « le plaisir sexuel poursuivi en tant que tel (p. 32) ». Contrairement à d'autres historiens ${ }^{12}$, il refuse d'appréhender les années 1920 comme celles d'une « révolution sexuelle » parce que celle-ci n'aurait pas été spontanée, mais dictée par le haut. L'auteur a conscience de l'usage discutable de cette notion de révolution sexuelle puisqu'il la place entre guillemets. Cependant, il l'introduit pour d'autres périodes, dont deux révolutions qui se suivraient en l'espace de quarante ans : les années 1960-1970, puis les années 1990, avec une perestroïka servant de pivot aux années 1990. La métaphore des " changements dynamiques sous la glace » qu'il choisit pour caractériser les années 1960-197013 est sans doute plus heureuse, car elle illustre parfaitement comment une libération des pratiques ne s'est pas traduite par une libération de la parole, du fait de l'immobilisme brejnévien. Si les nouvelles sources trouvées par Ira Roldugina, et l'analyse qu'elle en donne, pour les années 1920 témoignent que des homosexuels se sont sentis légitimés par la décriminalisation de leurs désirs pour faire entendre leur voix ${ }^{14}$, de quelle « révolution sexuelle », mise ou non entre guillemets, peut-il bien s'agir quand on écrit sur les années de la stagnation, quand le cinéaste Sergej Paradžanov par exemple peut être incarcéré malgré sa notoriété internationale ? Si libéralisation de la parole il y a, elle apparaît durant la perestroïka, mais n'est-elle pas rapidement supplantée par la commercialisation du sexe ? Comme le note finement Dan Healey, cette commercialisation produit une abondance d'images érotiques dont la société russe s'est offusquée tout en s'en délectant. À juste titre, il note que pour définir pudiquement l'homosexualité, l'expression d'orientation dite « non traditionnelle » commence à faire fortune dès les années 1990. Or, l'emploi d'une telle expression n'inviterait-il pas à nuancer, sinon à remettre en question, l'affirmation de l'historien selon laquelle les années 1990 seraient le théâtre d'une « révolution discursive "? C'est heureux, des questions restent encore ouvertes : la notion de « révolution sexuelle » chère à Wilhelm Reich constitue-t-elle ou non une catégorie d'analyse opérante? Si oui, pour quelle période l'est-elle plutôt que pour telle autre ? Quelles formes discursives propres prend-elle ?... Les recherches à venir sur l'URSS et la Russie permettront sans doute d'y répondre. « Comment 
peut-on faire l'histoire de l'homosexualité russe ? » : Dan Healey adapte et prend à son compte la question de David Harperin ${ }^{15}$ pour proposer de nombreuses et très riches pistes de recherche.

1 - Les Cahiers du Monde russe n'adoptant pas l'écriture inclusive, la recension a été adaptée en conséquence.

2 - Dan Healey, Homosexual Desire in Revolutionary Russia : The Regulation of Sexual Dissent, Chicago : The University of Chicago Press, 2001 ; Uladzimir Volodzin, Queer History of Belarus in the Second Half of the 20th Century : A Preliminary Study, Minsk, 2016. 3 - Adi Kuntsman, « With a Shade of Disgust : Affective Politics of Sexuality and Class in Memoirs of the Stalinist Gulag », Slavic Review, 68 (2), 2009.

4 - Lev Samojlov, Perevërnutyj mir, SPb. : FARN, 1993 [Le Monde sens dessus dessous]. 5 - Pour une analyse de ce que recouvre le terme russe «oglaska », cette crainte d'une divulgation publique de l'homosexualité, qu'une opinion publique entacherait d'un « jugement moral » : Arthur Clech, «Between the Labor Camp and the Clinic : Tema or the Shared Forms of Late Soviet Homosexual Subjectivities », Slavic Review, 77 (1), 2018, p. 6-29

6 - Oleg Kharkhordin, The collective and the individual in Russia : Study of practices, Berkeley : University of California Press, 1999.

7 - Arthur Clech, «Des subjectivités homosexuelles à l'époque soviétique tardive : entre solidarités et culture du soupçon », thèse de doctorat soutenue à l'École des hautes études en sciences sociales, Paris, 2018.

8 -Françoise Daucé et Elisabeth Sieca-Kozlowski, eds., Dedovshchina in the Post-Soviet Military. Hazing of Russian Army Conscripts in a Comparative Perspective, with a Foreword by Dale Herspring, Stuttgart : Ibidem- Verlag (Collection Soviet and Post-Soviet Politics and Society), 2006.

9 - Anon, «International », p. 60 cité comme tel dans Dan Healey, Russian Homophobia From Stalin To Sochi, New York : Bloomsbury, 2018, p.102.

10 - Voir notamment : Arthur Clech, « Des subjectivités homosexuelles dans une URSS multinationale », Le Mouvement Social, 260 (3), P. : Les Éditions de la découverte, 2017, p. 91-110 ; «Between the Labor Camp and the Clinic... », Slavic Review, 77 (1), 2018, p. 6-29 (dont une version révisée vient d'être publiée dans Richard Mole, ed. Soviet and Post-Soviet sexualities, Routledge, 2019, p. 32-55), et pour des analyses plus complètes : Clech, « Des subjectivités homosexuelles à l'époque soviétique tardive... ».

11 - Simon Karlinsky, « Russia's Gay Literature and Culture : The Impact of the October Revolution» in M.B. Duberman, M. Vicinus, G. Chauncey, eds., Hidden from History: Reclaiming the Gay and Lesbian Paste, New York : New American Library, 1989, p. 348-364.

12 - Gregory Carleton, Sexual Revolution in Bolshevik Russia, Pittsburgh : University of Pittsburgh Press, 2005.

13 - Dan Healey, «La révolution sexuelle en URSS : des changements dynamiques sous la glace » in Alain Giami et Gert Hekma, éds., Révolutions sexuelles, P. : la Musardine, 2015, p. 296-297.

14 - Ira Roldugina, « 'Whay are we the people we are' Early Soviet homosexuals from the first-person perspective : new sources on the history of homosexual identities in Russia », in Mole, ed., Soviet and Post-Soviet sexualities, initialement publié en russe : «"Počemu my takie ljudi?" : Rannesovetskie gomoseksualy ot pervogo lica : novye istočniki po istorii gomoseksual'nyh identičnostej v Rossii », Ab Imperio 2, 2016, p. 183-216.

15 - David Halperin, How to Do the History of Homosexuality, Chicago : The University of Chicago Press, 2002.

\section{Arthur Clech}

